\title{
Measurement of deformation rate in nasal septum deviation by three-dimensional computer tomography reconstruction and its application in nasal septoplasty endoscopic surgery
}

\author{
GUI YANG $^{1}$, HAILIANG ZHAO ${ }^{1}$, PENG WANG $^{1}$, XIAODONG HAN ${ }^{1}$, \\ XINYU ZAO ${ }^{1}$, ZHIXIAN LIU ${ }^{1}$, SHUQI QIU ${ }^{1-3}$ and ZHIQIANG LIU ${ }^{2,3}$ \\ ${ }^{1}$ Rhinology Department; ${ }^{2}$ Immunology Research Laboratory; ${ }^{3}$ Immunology and Allergy Laboratory, \\ Longgang ENT Hospital, Shenzhen, Guangdong 518172, P.R. China
}

Received March 10, 2016; Accepted March 3, 2017

DOI: $10.3892 /$ etm.2017.4644

\begin{abstract}
Nasal septum deviation (NSD) typically occurs following otorhinolaryngologic surgery. However, there is a lack of biomechanical parameters able to accurately evaluate the severity of NSD. The present study aimed to determine whether the deformation rate (DR) is associated with visual analogue scale (VAS) and nasal airway resistance (NAR), and to evaluate the application of DR measurements in nasal septoplasty endoscopic surgery. In the present clinical trial, a total of 30 patients with NSD were enrolled, and DRs were calculated prior to surgery by three dimensional computer tomography (3D-CT) reconstruction techniques combined with mechanical analysis. The distribution of stress lines at the nasal septum deviation site was evaluated prior to operation. Following nasal septoplasty endoscopic surgery, pre and postoperation scores for VAS and NAR were compared. The results demonstrated that DR was significantly correlated with preoperational NAR $(r=0.534)$ and VAS scores $(r=0.397)$. According to preoperative CT measurements of NSD, DR and biomechanical properties, selective excision was performed to remove core areas of stress. It was observed that postoperative DR, NAR and VAS scores were significantly lower (all $\mathrm{P}<0.01$ ) than those measured preoperation. Furthermore, over a follow-up period of 3 months, 23 cases (73.1\%) were cured and 7 cases $(23.3 \%)$ exhibited improvements. These results indicate that preoperative measurement of septum DR by 3D-CT reconstruction techniques may be important in determining the specific surgical approach of nasal septoplasty required.
\end{abstract}

Correspondence to: Professor Zhiqiang Liu, Immunology Research Laboratory, Longang ENT Hospital, 3004 Longgang Avenue, Longgang, Shenzhen, Guangdong 518172, P.R. China E-mail: liuzhiqiang05312438@126.com

Key words: nasal septum deviation, deformation rate, septoplasty, endoscopic surgery

\section{Introduction}

Nasal septum deviation (NSD) involves displacement of the nasal septum and typically occurs following otorhinolaryngologic surgery (1). Severe cases of NSD may be treated with a surgical procedure known as septoplasty. Based on the biomechanical characteristics of NSD, a novel surgical procedure termed nasal endoscopic three minus septoplasty has been developed by Wang et al (2). This modified version of septoplasty has so far been shown to be safe and effective at treating NSD, however it lacks the ability to measure preoperative biomechanical parameters required to evaluate the severity of NSD.

The deformation rate (DR) is defined as a change in tissue structure induced by an external force (3). During development and growth of the nasal septum, or in the process of ossification, imbalanced growth between the nasal bones or between the bones and cartilage may lead to nasal septum malformation and/or deviation, leading to formation of an apophysis at the nasal crest and/or junction (4). This process represents the elastic strain model of NSD. Due to the technical limitations of detection methods, few previous studies have successfully measured preoperative biomechanical parameters of NSD (2). However, due to the development of clinical three dimensional computer tomography (3D-CT) reconstruction techniques (5), it is now possible to measure and consult biomechanical parameters prior to nasal endoscopic septoplasty. DR, which is a key biomechanical parameter of the elastic strain model (2), may be calculated using 3D-CT reconstruction techniques. Therefore, using 3D-CT reconstruction techniques to measure the DR of the nasal septum at maximum deviation sites, the present study aimed to determine whether DR is associated with visual analogue scale (VAS) and nasal airway resistance (NAR) $(2,6)$, and to evaluate the application of DR measurements in nasal septoplasty endoscopic surgery.

\section{Materials and methods}

Clinical materials. The current study was conducted in 30 patients presenting with symptoms of NSD, who received nasal septoplasty endoscopic surgery in Longgang ENT Hospital (Shenzhen, China) between May 2014 and May 2015. 
The patient cohort included 21 males and 9 females and mean age was $35.2 \pm 12.3$ years. Patients exhibited mild to moderate clinical symptoms of nasal obstruction, including nasal hemorrhage, headache, nasal discharge, sneezing, snore and breathing through the mouth. VAS score was recorded by measuring the degree/strength of the symptoms to provide a measurable continuum as previously described $(2,7)$. NAR was measured using an NR6 nasal airway resistance dynamometer (GM Instruments Ltd., Kilwinning, UK) according to the manufacturer instructions. The mean VAS and NAR scores were $6.97 \pm 1.69$ and $1.36 \pm 0.30 \mathrm{kPa} . \mathrm{s}^{\mathrm{L}} \mathrm{L}^{-1}$, respectively. Patients suffering from nasal sinusitis or nasal polyp, granulomatous diseases of the nose or nasal masses, or with a past history of nasal surgery were excluded from the current study. The current study was approved by Ethics Committee of Longgang ENT Hospital and all patients provided written informed consent prior to participation in the study.

Preoperative 3D-CT reconstruction of the nasal septum. Preoperative 3D-CT scanning of nasal sinuses was performed in all patients using a Philips Brilliance 16-slice spiral CT (Philips Medical Systems B.V, Eindhoven, The Netherlands), to reconstruct coronal and/or axial nasal septum structures as original scanning image data. The 3D-CT scan ranged from the top of the frontal sinus to the bottom of hard palate. The scanning conditions were as follows: Voltage, $120 \mathrm{kV}$; current, $0.25 \mathrm{amp}$ slice thickness, $1 \mathrm{~mm}$ and layer spacing, $-1 \mathrm{~mm}$. The bone window settings were as follows: Window width, $2000 \mathrm{Hu}$ and window level, $200 \mathrm{Hu}$. The soft-tissue window settings were as follows: Window width, $350 \mathrm{Hu}$; and window level, $40 \mathrm{Hu}$. Scanning data were transferred to a Philips Extended Brilliance Workspace 3.5 workstation (Philips Medical Systems B.V.) and processed using a CT Viewer (Philips Medical Systems B.V.) for multiplanar reconstruction. Original soft tissue images were used to create $3 \mathrm{D}$ reconstruction models using a Sinus Trachea protocol (provided with workstation) and following reconstruction tissues adjacent to the nasal septum on the images were processed to expose the nasal septum. The position and lengths of four stress lines [as described by Wang et al (2)], the maximal deformation side of flexure and the original vertical length of the nasal septum were measured based on 3D and coronal images. The $3 \mathrm{D}$ reconstruction images were marked with the positioning and lengths of four stress lines: First line, between the quadrangular cartilage and the medial crus of the greater alar cartilage; second line, between the cartilage and vomer bone; third line, between the cartilage and the perpendicular plate of the ethmoid; fourth line, between the quadrangular cartilage and the nasal bone (Fig. 1).

Biomechanical analysis of the DR at the NSD site. The stress within deviated nasal septum bone is generally formed by flexural deformation. According to mechanical principles, the extent of stress is in direct proportion with the distance from the upper and lower boundaries to the central axis, with increased distance to the central axis resulting in greater stress $(2,8)$. The value of DR is equal to the maximal flexural deformation (a) minus the original length (b) divided by the original length (b), i.e., $D R=(a-b) / b$. In the current study, the deformed length of the nasal septum was defined as the maximal side of
Table I. Correlation analysis of DR1, NAR1 and VAS1.

\begin{tabular}{lccc}
\hline Item & DR1 & NAR1 & VAS1 \\
\hline DR1 & & & \\
$R$ & 1 & $0.397^{\mathrm{a}}$ & $0.534^{\mathrm{b}}$ \\
$\mathrm{P}$ & & 0.030 & 0.002 \\
NAR1 & & 1 & \\
$\mathrm{R}$ & & & 0.359 \\
$\mathrm{P}$ & & & 0.051 \\
VAS1 & & \\
$\mathrm{R}$ & & & \\
$\mathrm{P}$ & & & \\
\hline
\end{tabular}

${ }^{\mathrm{a}} \mathrm{P}<0.05,{ }^{\mathrm{b}} \mathrm{P}<0.01, \mathrm{n}=30$. DR1, preoperative elastic strain value; NAR1, preoperative nasal airway resistance; VAS1, preoperative visual analogue scale; R, Pearson correlation coefficient; P, P-value.

flexure (Fig. 1, indicated by the letter a). The strained side of the deformed septum cartilage was potentially caused by the nasal bone (upper side, a1), vomer and nasal crest of maxilla (lower side, a2). The original length was defined as the original vertical length of the maximal flexural side (Fig. 1, indicated by the letter $\mathrm{b}$ ). The following formula was used to calculate the $\mathrm{DR}$ value: $\mathrm{DR}=(\mathrm{a}-\mathrm{b}) / \mathrm{b}$.

Surgical methods. All surgeries were performed using a Storz nasal endoscope (KARL STORZ GmbH \& Co., KG, Tuttlingen, Germany) and surgical monitoring system (Medtronic, Minneapolis, MN, USA). According to assessments of disease severity, general conditions and psychological status, 30 patients were administered general anesthesia by an anesthetist according to standard clinical practices and placed in a supine position. The nasal skin was incised to expose the perichondrium by Killian incision. Structures from the superior and posterior side of the lamina mediana and Vomer to the inferior and posterior structures at the nasal bottom, including the nasal septum bottom, Vomer and nasal crest of maxilla were separated. On the posterior side, within 1-2 $\mathrm{mm}$ of the first skin and mucous incision, two incisions with an interval of $2 \mathrm{~mm}$ were made in parallel through the cartilage of the nasal septum. Cartilage between the two incisions was removed and contralateral mucous cartilage membrane was separated using a nasal endoscope, in order to separate the mucous cartilage membrane from the nasal septum cartilage, as previously described (9). A double heads nasal septum elevator (Guangzhou in LaCrosse Medical Devices Co., Ltd., Guangzhou, China) was used to compress the junction between the nasal septum cartilage and lamina mediana, as the connection between the nasal septum cartilage, Vomer ridge at the basal base and nasal crest of maxilla. Pressure was applied to dislocate the nasal septum cartilage and separate the nasal septum cartilage at the front, posterior and inferior sides. Based on preoperative $3 \mathrm{D}$ reconstruction data, selective excision was conducted to remove the core areas of stress and eliminate the stress between the septum cartilage and sclerostin (2). Images of the bilateral nasal cavities were obtained by endoscopic examination immediately before and after surgery. 
Table II. Comparison of pre and postoperative DR, NAR and VAS values.

\begin{tabular}{lccc}
\hline Item & DR & NAR, kPa.s.L-1 & VAS score \\
\hline Preoperative & $0.148 \pm 0.105^{\mathrm{a}}$ & $1.36 \pm 0.30^{\mathrm{a}}$ & $6.97 \pm 1.69^{\mathrm{a}}$ \\
3 months after surgery & $0.017 \pm 0.015$ & $0.27 \pm 0.14$ & $1.37 \pm 0.89$ \\
$\mathrm{t}$ & 6.98 & 21.96 & 16.75 \\
P-values & $<0.01$ & $<0.01$ & $<0.01$ \\
\hline
\end{tabular}

$\mathrm{n}=30$. DR, deformation rate; NAR, nasal airway resistance; VAS, visual analogue scale; t, t-value; $\mathrm{P}, \mathrm{P}-\mathrm{value}$. ${ }^{\mathrm{a}} \mathrm{P}<0.01$ compared with 3 month after surgery.

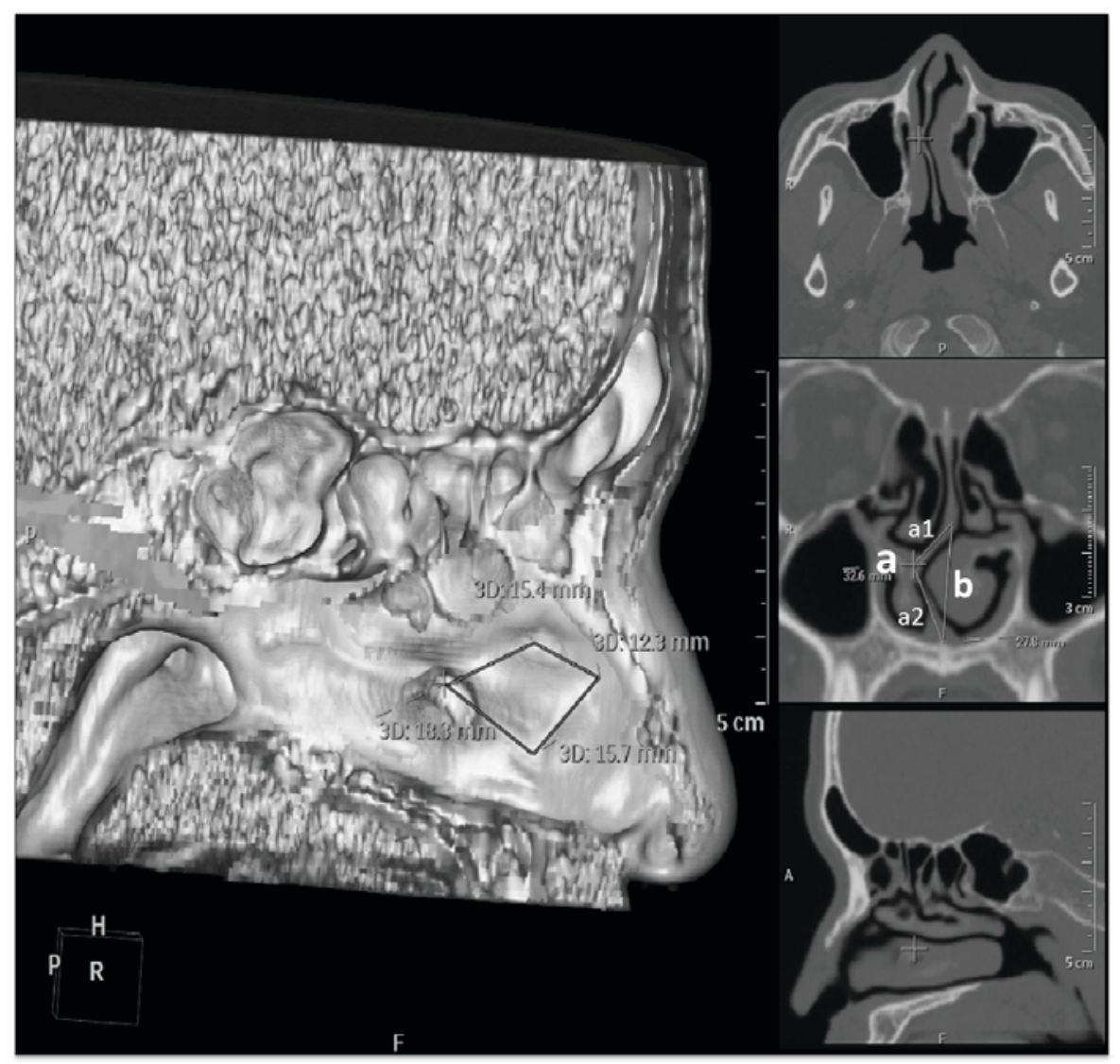

Figure 1. Representative image of three dimensional computer tomography reconstruction. The position and lengths of four stress lines are indicated (black quadrilateral). The coronal scan (right panel) also indicates the maximal side of flexure (a), the strained side of the deformed septum cartilage was potentially caused by the nasal bone (upper side, a1), vomer and nasal crest of maxilla (lower side, a2) and the original vertical length (b).

Therapeutic evaluation. Patients were followed up 3 months after surgery to evaluate NSD-related symptoms (10). As a subjective evaluation standard, VAS was used to identify the severity of nasal obstruction caused by lesions of the nasal cavity. NAR was assessed using an NR6 nasal airway resistance dynamometer (GM Instruments Ltd.) as an objective evaluation parameter of nasal ventilation function. Data were collected from patients before and 3 months after the operation.

Statistical analysis. Data are presented as the mean \pm standard deviation. Data from the preoperative assessment of VAS (VAS1), preoperative NAR (NAR1), postoperative VAS (VAS2) and postoperative NAR (NAR2) were analyzed using a paired t test and Pearson correlation analysis. SPSS 19.0 software (IBM SPSS, Armonk, NY, USA) was used for statistical analysis and $\mathrm{P}<0.05$ was considered to indicate a statistically significant difference.

\section{Results}

Reconstructed 3D images of the nasal septum. Among the 30 patients of NSD enrolled in the present study, there were 24 cases of ' $C$ ' deviation, 6 cases of ' $S$ ' deviation, 18 cases of crest elevation and 3 cases of nasal process deviation (7). A total of 19 patients exhibited deviation at the superior position and 7 patients exhibited deviation at the caudal end. Following 3D reconstruction and excision, distinct 3D images 


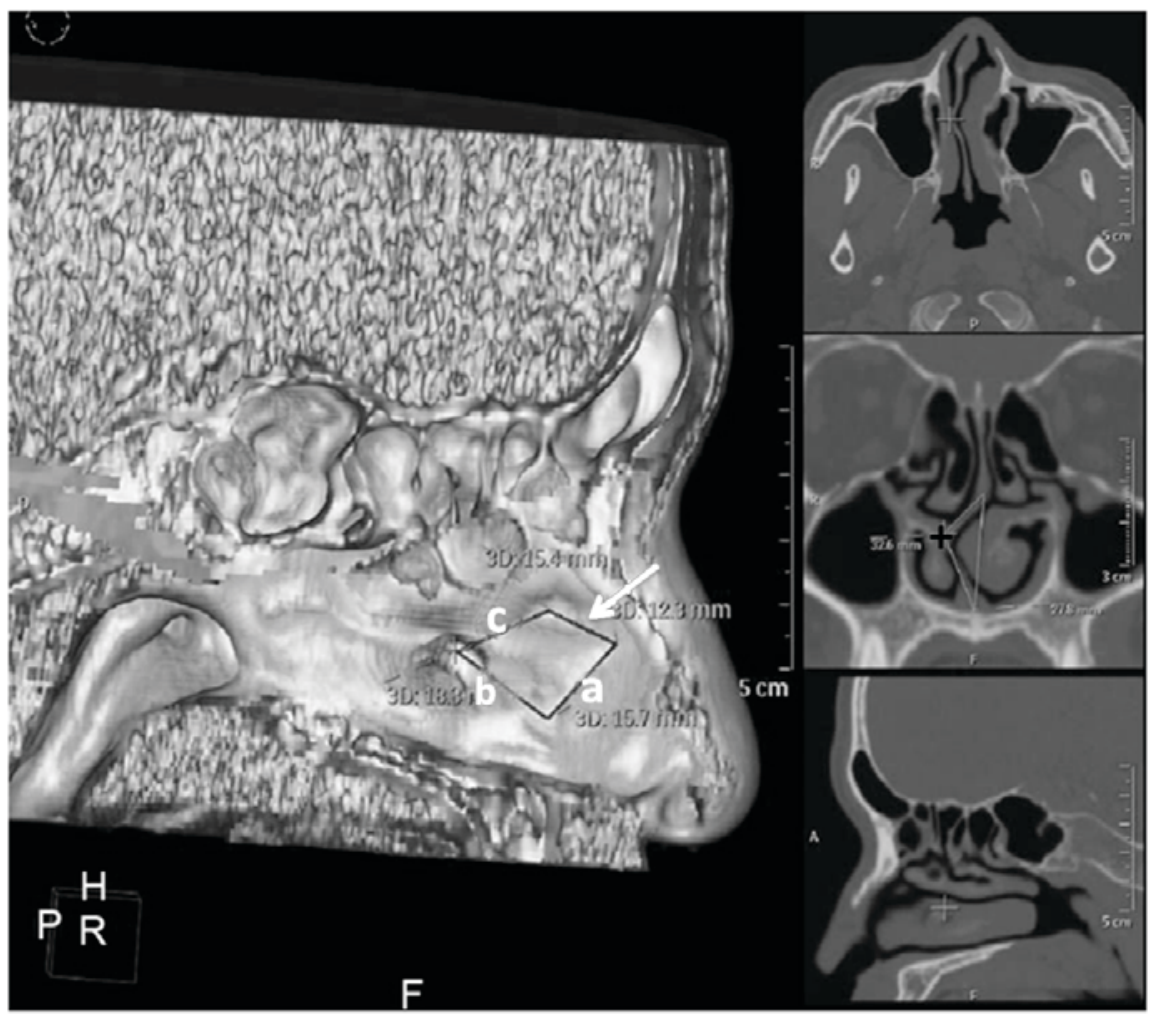

Figure 2. Representative image of three dimensional computer tomography reconstruction. Marked right deviation of the nasal septum is evident at the first (a), second (b) and third (c) stress lines. The fourth stress line is also indicated (white arrow). The intersection between the second and third stress lines is the most obvious deviation site (+).

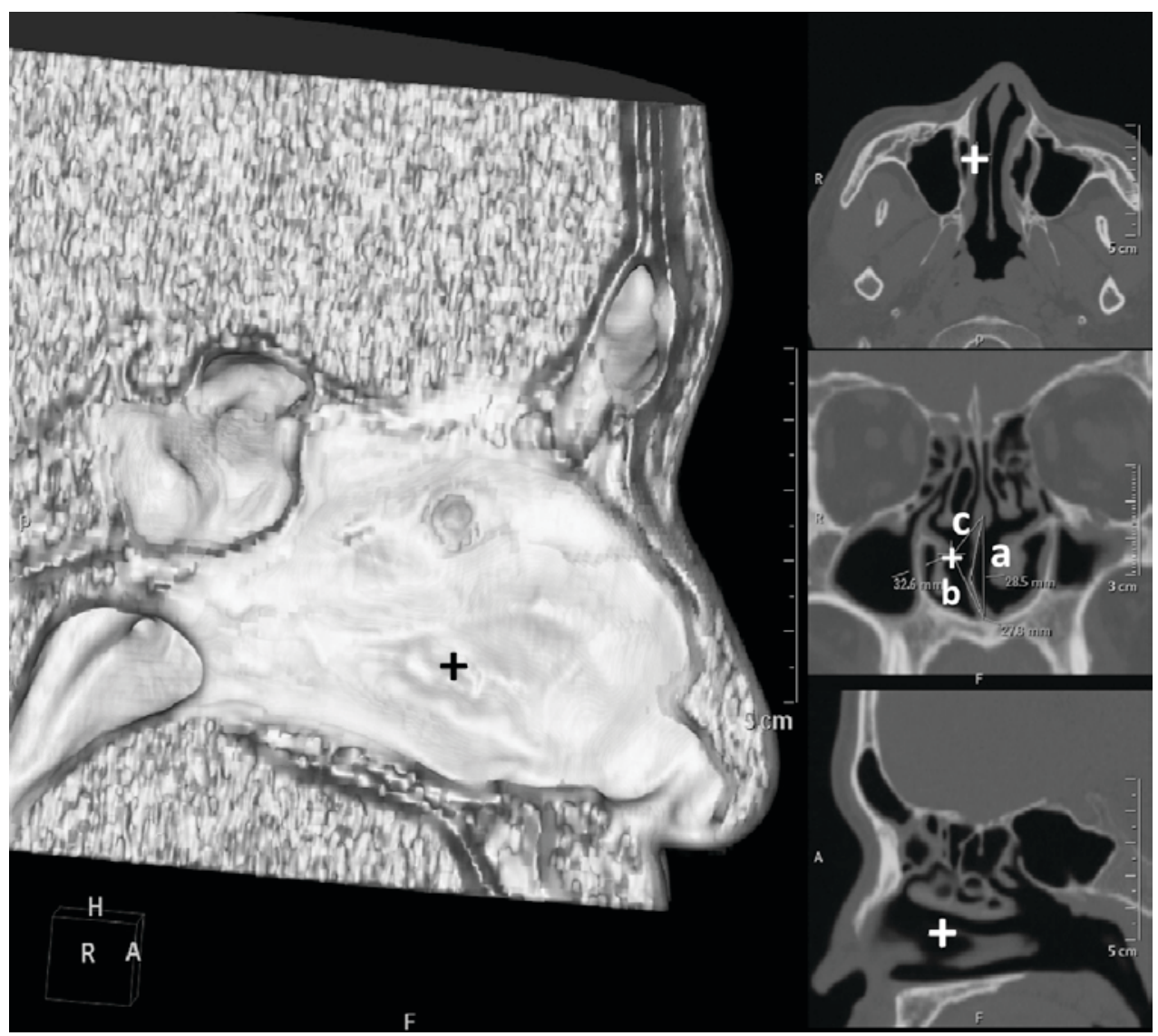

Figure 3. Representative image of three dimensional computer tomography reconstruction. Image presents the most severe region of the original deviation $(+)$, the original vertical length (a), the primary maximum curved edge of nasal septum deformation (b), and the maximum edge of membranous deviation following correction (c). 
A

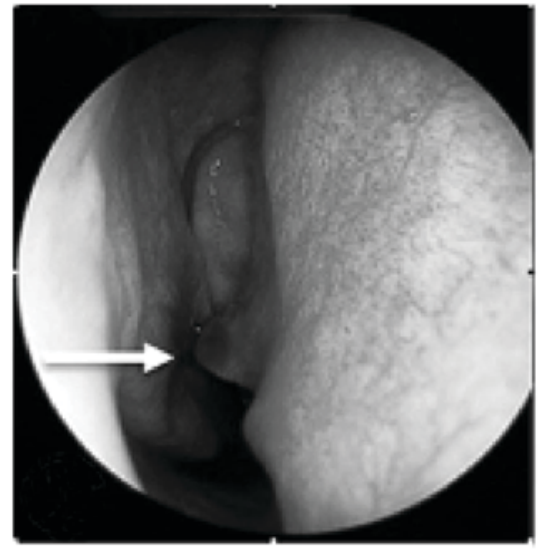

C

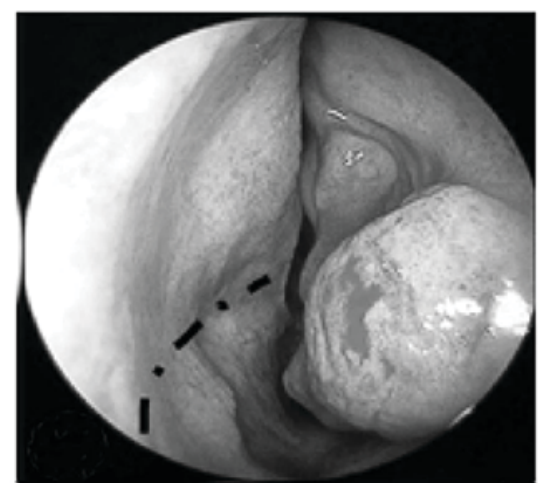

$\mathrm{B}$
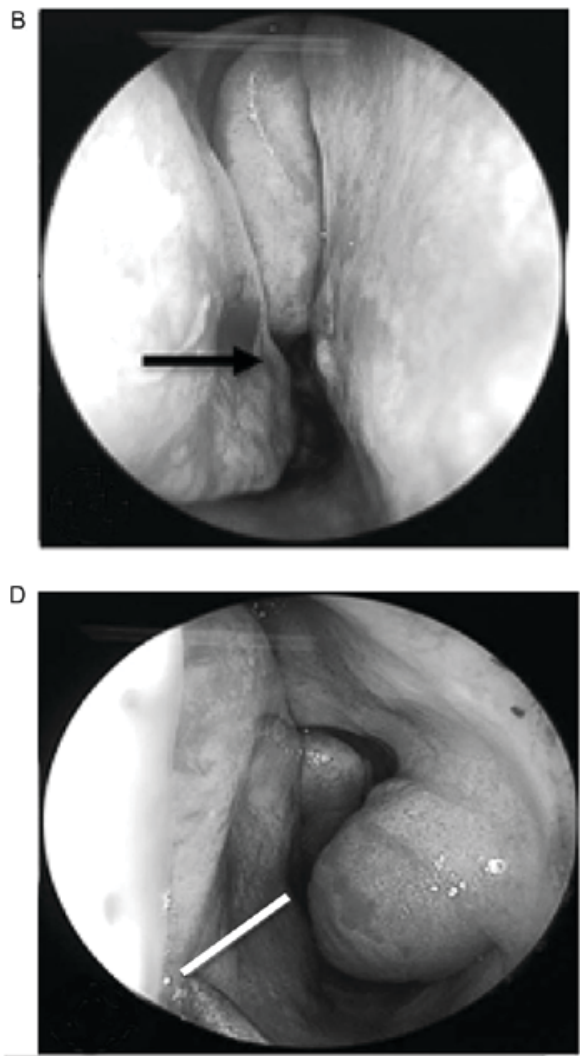

Figure 4. Representative images of endoscopic examination before and after surgery. (A) The right nasal cavity prior to surgery containing the most obvious deviation site (white arrow). (B) The right nasal cavity following surgery, in which stress has been relieved (black arrow). (C) The right nasal cavity prior to operation with the second stress line indicated (dotted black line). (D) The right nasal cavity after surgery, in which the second stress line has been relieved (solid white line).

of the nasal septum indicating the shape and site of deviation were obtained. For instance, the coronal and sagittal 3D-CT images from one patient presented with right deviation of the nasal septum, located at the first [Fig. 2; a], second [Fig. 2; b] and third [Fig. 2; c] stress lines. However, deviation was not detected at the fourth stress line (Fig. 2; black arrow). The most marked deviation site was located at the junction between the quadrilateral cartilage and lamina mediana, at the borderline between the second and third stress lines (Fig. 2, '+' symbol). Using the coronal plane of this point, values of deformation and original length were determined.

Preoperative 3D reconstruction images successfully identified the following: The most severe region of the original deviation (Fig. 3, '+'); the original vertical length (Fig. 3; a), the primary maximum curved edge of NSD (Fig. 3; b); and the maximum edge of membranous deviation (Fig. 3; c). Based on these observations, regions of stress were determined. According to preoperative $3 \mathrm{D}$ reconstruction images and the calculated DR $(0.148 \pm 0.105)$, pointed intraoperative relief of nasal septum stress was performed. Preoperative 3D-CT reconstruction images were reviewed 3 months after surgery. Images of the bilateral nasal cavities obtained by endoscopic examination immediately before and after surgery (Fig. 4) confirmed that the state of the nasal septum at the time of operation was consistent with the data provided by preoperative $3 \mathrm{D}$ reconstruction images.

Correlation analysis of preoperative DR1, NAR1 and VAS1. Potential correlations between DR1, NAR1 and VAS1 were evaluated by Pearson correlation analysis (Table I). DR1 was significantly correlated with VAS1 $(\mathrm{P}=0.002)$ with a Pearson correlation coefficient of 0.534 . The Pearson correlation coefficient between DR1 and NAR1 was 0.397, indicating a significant association between DR1 and NAR1 $(\mathrm{P}=0.03)$. NAR1 was not significantly correlated with VAS1.

Postoperative follow-up and clinical efficacy. Based on preoperative $3 \mathrm{D}$ reconstruction images and DR analysis, a general nasal septoplasty endoscopic operation was performed, whereby relaxing surgery was conducted on the nasal septum. During a 3 month follow-up period, 23 cases (73.1\%) were cured and 7 cases $(23.3 . \%)$ had improved. The postoperative DR2, NAR2 and VAS2 values 3 months after surgery were all significantly decreased compared with the preoperative DR1, NAR1 and VAS1 values, respectively $(\mathrm{P}<0.01$; Table II). During this time, no complications occurred.

\section{Discussion}

NSD is a prevalent clinical condition, with severe cases typically leading to breathing difficulties, nasal obstruction, nasal hemorrhage and headache. Fischer and Gubisch (11) documented that NSD was the primary cause of obstructive disorders of the nasal valve area and may interfere with the elimination of inflammatory mediators. NSD correlates with the pathogenesis of allergic rhinitis and is a major risk 
factor of obstructive sleep apnea syndrome (12). Severe NSD generally requires the surgical treatment, usually septoplasty, as it is considered to be the most effective method of treating NSD (13). The fundamental purpose of septoplasty is to relieve the clinical symptoms induced by NSD and the surgery may also be used to treat concurrent disorders such as nasal sinusitis (14). In nasal septum reconstruction, the traditional procedure of submucous septectomy has been developed into the more novel techniques of nasal submucous septorhinoplasty, used in cases requiring septal cartilage alteration, and nasal endoscopic three minus septorhinoplasty (15). These novel procedures preserve the nasal supporting structures and reduce complications; however, they are more complicated than the traditional technique, and require higher operating skills and sufficient awareness of the deviation site, stress area and biomechanical parameters to evaluate the levels of NSD prior to operation. Furthermore, Akoglu et al (16) suggested that any substantial bony and mucosal enlargements in the anterior and middle segments of the inferior turbinate should be unaltered, due to their supporting roles in turbinate reduction during septoplasty. Few studies of operative techniques have involved the assessment of the NSD elastic stress area and preoperative biomechanical parameters. Biomechanical principles are extensively applied in orthopedics and other fields $(17,18)$. Despite a lack of muscle tissue and the influence of other mechanical factors, the anatomical structure of the nasal septum can be generally viewed as an elastic mechanical model that conforms to biomechanical rules (19). During the embryonic phase of development, the nasal septum is composed of different cartilages (20). As development of the skull occurs prior to maturation of the nasal septum, the upper and bottom boundaries of nasal septum are fixed, and any imbalanced growth between the bone and cartilage and/or bone and bone during development and ossification produces strain and stress variation (21). This variation is predominantly concentrated in the junctions between the thin nasal septum cartilage and peripheral bones, eventually leading to the development of nasal deformity or deviation and apophysis at the crest of the nasal septum $(22,23)$. In biomechanical terms, the strain and stress that occurs in NSD is consistent with flexural load, and the DR represents the ratio between the septum deformation and original septum size, reflecting the magnitude of strain and stress in the nasal septum. Therefore, analysis of the DR is an important factor to consider for nasal submucous septorhinoplasty, as the results of the present study indicated that according to the evaluation of DR, relieving strain and stress in NSD is key for successful correction of NSD.

In the present study, the general morphology, deviation site, maximum point of deviation and stress lines of the nasal septum were accurately identified by $3 \mathrm{D}-\mathrm{CT}$ reconstruction images prior to surgery. In addition, assessment of the DR enabled accurate selection of the correct procedure and the operating site for stress relief, resulting in successful septorhinoplasty based on three line stress relaxation. In classical nasal septum reconstruction, the left Killian incision aims to penetrate at the borderline between the skin and mucus; however in practice, this incision is complicated and should be selected and modified based on preoperative evaluation of
3D-CT images and DR. Preoperative analysis in the current study also identified a fourth stress line, as a potential region to excise a fraction of cartilage and bone during surgery. The actual sites of excision were ultimately determined by specific measurements and observations during surgery, in order to maximally preserve the supporting structures. However, the results of the current study are limited due to the relatively small sample size. Therefore, future large sample studies are warranted to validate the use of DR assessment in determining the strain area in NSD.

In conclusion, 3D-CT reconstruction techniques and subsequent mechanical analysis were used in the present study to calculate the DR of NSD prior to surgery. The distribution of stress lines at the site of NSD were also evaluated prior to surgery. It was determined that the DR of NSD was significantly associated with preoperative NAR and VAS score. Therefore, preoperative measurement of the septum DR by $3 \mathrm{D}-\mathrm{CT}$ reconstruction techniques may be important in determining the specific surgical approach required for nasal septoplasty.

\section{Acknowledgements}

The present study was supported by the Natural Science Foundation of China (grant nos. 31400856, 81571790 and 81501573), the Longgang District Science and Technology Plan (grant no. YLWS20140609120004346), the Key Laboratory Project of Shenzhen (grant no. ZDSYS201506050935272) and the Innovation of Science and Technology Commission of Shenzhen Municipality (grant no. JCYJ20140411150916749, JCYJ20160429091935720).

\section{References}

1. Schuman TA and Labadie RF: Concurrent nasal surgery and tympanoplasty in adults. Ear Nose Throat J 89: E28-E32, 2010.

2. Wang T, Han D, Zhang L, Zang H, Li Y and Liu C: A modified septoplasty with three high tension lines resection. Acta Otolaryngol 130: 593-599, 2010.

3. Atsumi A, Seo Y, Ishizu T, Nakamura A, Enomoto Y, Harimura Y, Okazaki T, Abe Y and Aonuma K: Right ventricular deformation analyses using a three-dimensional speckle-tracking echocardiographic system specialized for the right ventricle. J Am Soc Echocardiogr 29: 402-411,e2, 2016.

4. Hall BK and Precious DS: Cleft lip, nose, and palate: The nasal septum as the pacemaker for midfacial growth. Oral Surg Oral Med Oral Pathol Oral Radiol 115: 442-447, 2013.

5. Steinbacher DM: Three-dimensional analysis and surgical planning in craniomaxillofacial surgery. J Oral Maxillofac Surg 73 (12 Suppl): S40-S56, 2015.

6. Swoboda S, Thulesius HL and Jessen M: Paradoxical increase in nasal airway resistance after topical nasal decongestion-does it really exist? A retro- and prospective study. Clin Otolaryngol 41: 689-693, 2016.

7. Fokkens W, Lund V and Mullol J; European Position Paper on Rhinosinusitis and Nasal Polyps Group: EP3O5 2007: European position paper on rhinosinusitis and nasal polyps. A summary for otorhinolaryngologists. Rhinology 45: 97-101, 2007.

8. Takahashi R: The formation of the nasal septum and the etiology of septal deformity. The concept of evolutionary paradox. Acta Otolaryngol Suppl 443: 1-160, 1987.

9. Getz AE and Hwang PH: Endoscopic septoplasty. Curr Opin Otolaryngol Head Neck Surg 16: 26-31, 2008.

10. Thulesius HL, Cervin A and Jessen M: Can we always trust rhinomanometry? Rhinology 49: 46-52, 2011.

11. Fischer $\mathrm{H}$ and Gubisch W: Nasal valves-importance and surgical procedures. Facial Plast Surg 22: 266-280, 2006.

12. Georgalas C: The role of the nose in snoring and obstructive sleep apnoea: An update. Eur Arch Otorhinolaryngol 268: 1365-1373, 2011. 
13. Moore M and Eccles R: Objective evidence for the efficacy of surgical management of the deviated septum as a treatment for chronic nasal obstruction: A systematic review. Clin Otolaryngol 36: 106-113, 2011

14. Orlandi RR: A systematic analysis of septal deviation associated with rhinosinusitis. Laryngoscope 120: 1687-1695, 2010.

15. Lee JE, Jung HJ, Chang M and Jin HR: A novel wedge technique to correct the curved deviation of the cartilaginous nasal septum. Auris Nasus Larynx 41: 190-194, 2014.

16. Akoglu E, Karazincir S, Balci A, Okuyucu S, Sumbas H and Dağli AS: Evaluation of the turbinate hypertrophy by computed tomography in patients with deviated nasal septum. Otolaryngo Head Neck Surg 136: 380-384, 2007.

17. Obert L, Uhring J, Rey PB, Rochet S, Lepage D, Leclerc G, Serre A and Garbuio P: Anatomy and biomechanics of distal radius fractures: A literature review. Chir Main 31: 287-297, 2012 (In French).

18. Cardoso L and Weinbaum S: Changing views of the biomechanics of vulnerable plaque rupture: A review. Ann Biomed Eng 42: 415-431, 2014.
19. Moshirfar M, Edmonds JN, Behunin NL and Christiansen SM: Corneal biomechanics in iatrogenic ectasia and keratoconus: A review of the literature. Oman J Ophthalmol 6: 12-17, 2013.

20. Neskey D, Eloy JA and Casiano RR: Nasal, septal, and turbinate anatomy and embryology. Otolaryngol Clin North Am 42 193-205, vii, 2009.

21. Yilmaz NA, Cicekcibasi AE, Emlik D, Yilmaz MT, Keles B and Salbacak A: Morphometric analyses of the development of nasal cavity in human fetuses: An anatomical and radiological study. Int J Pediatr Otorhinolaryngol 74: 796-802, 2010.

22. Cantrell H: Limited septoplasty for endoscopic sinus surgery. Otolaryngol Head Neck Surg 116: 274-277, 1997.

23. VanLoosen J, VanZanten GA,Howard CV, Verwoerd-VerhoefHL, Van Velzen D and Verwoerd CD: Growth characteristics of the human nasal septum. Rhinology 34: 78-82, 1996. 